\title{
Forward genetics in Tribolium castaneum: opening new avenues of research in arthropod biology
}

\author{
Andrew D Peel
}

Address: Institute of Molecular Biology and Biotechnology (IMBB), Foundation for Research and Technology Hellas (FoRTH), Nikolaou Plastira 100, GR-70013 Iraklio, Crete, Greece. Email: apeel@imbb.forth.gr

\begin{abstract}
A recent paper in BMC Biology reports the first large-scale insertional mutagenesis screen in a non-drosophilid insect, the red flour beetle Tribolium castaneum. This screen marks the beginning of a non-biased, 'forward genetics' approach to the study of genetic mechanisms operating in Tribolium.
\end{abstract}

See research article http://biomedcentral.com/1741-7007/7/73

Much of our understanding of the genetic mechanisms operating in arthropods is derived from studies on the genetically tractable, and long established, laboratory model insect Drosophila melanogaster. However, despite the many advantages of using the Drosophila model system, it does have some inherent theoretical and practical limitations. Many of the traits that predispose Drosophila to laboratory study - for example, its small genome and developmental traits associated with its short generation time - are evolutionarily derived and/or atypical of many arthropods. As such, it has long been accepted that a greater depth of knowledge from a broader range of arthropods is required to gain a clearer understanding of the ancestry and evolution of arthropod developmental mechanisms. In addition, studies on arthropod species that exhibit morphological, physiological, behavioral or ecological traits absent in Drosophila are often a prerequisite to address a specific theoretical question or practical problem.

There has therefore been a pressing need to establish reliable and efficient tools for genetic manipulation in arthropod species that often possess larger genomes than Drosophila, or exhibit longer and less amenable life histories. Much progress has been made in recent years. The advent of reverse genetic techniques, most notably RNA interference (RNAi), has enabled the disruption of gene function in a wide range of arthropods. The increasing speed, and reduced cost, of DNA sequencing has meant that complete genome sequences (and/or expressed sequence tags, ESTs) are now available to the research community for an ever-increasing number of species. And now, in a paper published in BMC Biology, Trauner et al. [1] report another significant advance: the first large-scale insertional mutagenesis screen in a nondrosophilid arthropod, the red flour beetle Tribolium castaneum. Chemical and/or gamma-irradiation mutagenesis screens selecting for specific classes of mutant phenotype have been carried out before in Tribolium [2,3], as well as in the parasitic wasp Nasonia vitripennis [4]. However, the insertional mutagenesis screen reported by Trauner et al. [1] will facilitate, for the first time in a non-drosophilid arthropod, a large-scale and non-biased approach to the study of genetic mechanisms underpinning a diverse range of biological traits.

\section{The first large-scale insertional mutagenesis screen in a non-drosophilid arthropod}

Of the non-drosophilid arthropods currently under study, the beetle Tribolium castaneum is the most amenable to genetic manipulation and is rapidly becoming a model arthropod system. The Tribolium genome is fully sequenced, well aligned and available to the research community [5]. Reverse genetics, via RNAi, is highly efficient, being both systemic in nature and applicable to all life stages [6]. In addition, effective protocols have been developed for germline transformation and insertional mutagenesis that make use of a number of different transposable elements and dominant fluorescent marker genes [7-10]. Trauner et al. [1] have used this existing transgenic technology, and a strategy devised and tested previously [8], to undertake a large-scale insertional mutagenesis screen in $T$. castaneum, the first in a nondrosophilid arthropod.

The chemical and gamma-irradiation mutagenesis screens carried out previously in Tribolium identified many mutants that proved informative with respect to specific processes, such as the genetic mechanisms controlling the development and diversification of body segments $[2,3]$. However, the absence of dominant markers, coupled with insufficient balancer chromosomes (there is currently less than $40 \%$ genome coverage), made the characterization and maintenance of recessive mutants difficult on the scale necessary for large non-biased screens. The insertional mutagenesis screen carried out by Trauner et al. [1] has 
four important features that confer practicality of use on a large scale.

\section{Donor and helper strains}

By using two distinct transposons to establish stable 'donor' and 'helper' strains, the need for repetitive - and less efficient - egg injections to create new transgenic lines was avoided [8]. The donor strain carries the transposon (in this case derived from the lepidopteran piggyBac element) that is remobilized to produce new insertions, whereas the helper (or 'jumpstarter') strain carries the stably integrated source of transposase that is necessary to catalyze these remobilization events (in this case the Minos transposable element was used to stably integrate a source of piggyBac transposase). New transgenic lines were established simply by crossing the donor and helper strains, such that the piggyBac transposon and transposase were present in the same individual. The resulting new transposon insertions were then stabilized in the next generation by segregating away the helper element (that is, the piggyBac transposase).

\section{Dominant fluorescent markers}

Efficient identification of new transgenic lines and their subsequent stabilization and maintenance was achieved by using dominant fluorescent markers. Hybrid beetles competent for germline remobilization of the donor element were identified by their red and green fluorescent eyes, which resulted from the expression of enhanced green fluorescent protein (EGFP) from the piggyBac donor element and DsRed from the helper element. The fact that the $3 \mathrm{xP}_{3}$ universal promoter used to drive this eyerestricted expression has enhancer-trapping capabilities was exploited to identify those beetles in which remobilization of the donor element had actually occurred [7]. A donor strain was chosen in which the donor element is integrated into the 3 ' untranslated region of an actin gene [8], resulting in expression of EGFP in muscle tissue as well as in the eyes; in individuals where the donor element is remobilized away from this actin gene the green fluorescence in muscles is lost. Thus individual $\mathrm{F} 1$ beetles that retained green eye fluorescence but lacked green muscle fluorescence and red eye fluorescence could be easily selected to found new and stable transgenic lines.

\section{An optimized crossing scheme to identify new recessive mutant lines}

Although by far the most laborious phase of the screen, Trauner et al. [1] devised a crossing scheme for the identification of recessive mutant lines that did not require balancer chromosomes, that minimized the number of false positives while practically eliminating the chances of false negatives (that is, discarding true recessive mutant lines), and that still identified sufficient numbers of homozygous lethal, semi-lethal and sterile lines to make the screen worthwhile (see below and [1]).

\section{Simple identification of affected genes}

Mutagenesis via the physical insertion of a transposon, when combined with a fully sequenced genome [5], makes identification of the affected gene or genes relatively simple. Genomic sequence flanking the inserted transposon was obtained using a suite of PCR-based methods, with subsequent BLAST analysis usually identifying around the site of insertion a small number of candidates for the gene mutated or trapped.

Using this scheme, Trauner et al. [1] were able to generate and analyze more than 6,500 new piggyBac insertion lines, which identified 421 embryonic recessive lethal insertions, 75 embryonic recessive semi-lethal insertions and 8 recessive sterile insertions. This rate of generating recessive lethal mutations in $T$. castaneum was on a par with comparable insertional mutagenesis screens carried out previously in Drosophila. Of particular importance, embryonic homozygous lethal mutations exhibited a range of phenotypes in both morphological space and developmental time. Encouragingly, insertions within introns in two genes that have already been well studied Tc-Krüppel and Tc-maxillopedia - recapitulated, at least in part, the knockdown phenotypes previously generated by RNAi $[1,8]$.

The authors estimated that using this scheme one person could establish 150 recessive lethal strains in one year. While not yet efficient enough to attempt genome saturation, this number should increase with improvements to the mutating potential of donor elements (for example, via the use of insulator sequences or splice acceptor sites) and/ or the introduction of dominant marking systems that will allow the simultaneous determination of sex and identification of new insertions (for details see [1]). The screen also identified 505 lines exhibiting new enhancertrap patterns, which will be directly informative with respect to the developmental mechanisms operating in Tribolium.

Analysis of the chromosomal locations of 403 of the piggyBac insertions revealed that with the exception of a bias for reinsertion near the site of mobilization, insertions were well distributed throughout the Tribolium genome. As a result, the large number of embryonic recessive lethal and enhancer-trap lines generated by this and future screens will for the first time enable a non-biased approach to the study of Tribolium genetics.

\section{The advantage of a non-biased genetic approach to the study of arthropod biology}

The study of genetic mechanisms in most arthropods has been restricted to examining the homologs of genes with well-characterized roles in the experimentally amenable, but evolutionarily derived, fruit fly Drosophila melanogaster. This 'candidate gene approach' has proved informative. 
For example, it has revealed that developmental genes are broadly conserved across phylogenetically widespread and morphologically diverse arthropod species. It has suggested that the changes underpinning diversifications in arthropod morphology have occurred as much, if not more, via the 'rewiring' of existing genetic networks, and through the cooption of existing genes into new roles, than by the emergence of entirely novel genes.

However, the candidate gene approach has significant limitations. It overlooks those genes whose functions are not yet characterized in Drosophila, genes that obtained novel roles in the lineages leading to non-drosophilid species, as well as the fraction of genes that lost their ancestral roles (or were lost all together) in the lineage leading to Drosophila. Indeed, genome comparisons reveal that there are thousands of genes in both Drosophila and Tribolium that currently appear species specific (that is, no cross-species sequence similarity can be identified) [5]. This implies the existence of a significant number of novel genes, or genes that have diversified in function between the lineages, perhaps many of these associated with species-specific traits. Genome comparisons also show that in each lineage a small, but significant, number of ancestral gene families - as determined by their presence in other arthropod and vertebrate genomes - have been lost altogether [5].

An example of a gene that might have been overlooked by following a purely candidate gene approach is the Tribolium developmental gene mille-pattes [11]. An important role in Tribolium thoracic and abdominal segmentation for this highly unusual gene - four small peptides are translated from its polycistronic transcript was revealed by its appearance in an EST expression screen [11] (an alternative non-biased genetic resource available in Tribolium). A homologous gene, called tarsal-less ( $t a l$ ), is present in Drosophila. Although tal is expressed in a segmental pattern, tal mutants do not show any segmentation or homeotic phenotypes [12], and thus millepattes would not have been an obvious candidate for a role in Tribolium segmentation [12].

Many similar examples will no doubt arise as the lines established by Trauner et al. [1] are closely examined by the Tribolium research community: information on these lines can be found at the GEKU database [13], and all lines are freely available on request. Indeed, the first study using a line from this screen has already appeared in print. Kittelmann et al. [14] examined the new enhancer traps for lines exhibiting expression of EGFP in thoracic legs. The subsequent analysis of one such line identified a role for the Tribolium homolog of the Drosophila gene zinc finger homeodomain 2 (zfh2) in distal leg development as well as leg segmentation [14]. Once again, a purely candidate gene approach could not have led to this finding, as Drosophila zfh2 has no reported role in leg development [14].

\section{Future developments in Tribolium and beyond}

The ectopic misexpression of genes can offer important insights on function that complement data derived from RNAi knockdown experiments. With the generation of a large number of enhancer-trap lines, an ability to conditionally misexpress genes in temporally and spatially restricted domains in Tribolium draws nearer. This could potentially be achieved by engineering donor elements to be competent in site-specific recombination: the sitespecific integration system from phage phiC 31 has already been used successfully to modify existing transgenic lines in Drosophila and in the Mediterranean fruit fly Ceratitis capitata $[15,16]$. This strategy would use a stably integrated enhancer-trapping donor element as a 'landing pad' for the site-specific integration of a gene construct whose transcription would then come under the control of the same enhancer(s) driving the original enhancer trap EGFP expression pattern. If the development of binary expression systems - such as the yeast-derived GAL4/UAS system widely used in Drosophila - proves successful in Tribolium, such a strategy could be used to establish a variety of stable (GAL4) driver lines, that could then be crossed to transgenic (UAS) effector lines in order to temporally and or spatially misexpress genes.

As all the genetic components used are species nonspecific, large-scale insertional mutagenesis screens analogous with that carried out by Trauner et al. [1] can potentially be extended to other arthropods in which large-scale crossing schemes and the maintenance of transgenic lines is feasible. Indeed, significant progress towards this end is currently being made in the amphipod crustacean Parhyale hawaiensis, in which transgenic methods have already been used to conditionally misexpress the homeotic gene Ultrabithorax ([17] and M Averof, personal communication). The establishment of a number of additional model arthropod systems that are amenable to genetic manipulation promises to open many new avenues of research. The advent of forward genetics in Tribolium signals the start of a new and exciting phase in the study of arthropod biology.

\section{Acknowledgements}

I would like to thank Z Kontarakis and M Averof for their helpful comments on the manuscript.

\section{References}

1. Trauner J, Schinko J, Lorenzen MD, Shippy TD, Wimmer EA, Beeman RW, Klingler M, Bucher G, Brown SJ: Large-scale insertional mutagenesis of a coleopteran stored grain pest, the red flour beetle Tribolium castaneum, identifies embryonic lethal mutations and enhancer traps. BMC Biol 2009, 7:73.

2. Beeman RW, Stuart JJ, Haas MS, Denell RE: Genetic analysis of the homeotic gene complex (HOM-C) in the beetle Tribolium castaneum. Dev Biol 1989, 133:196-209.

3. Sulston IA, Anderson KV: Embryonic patterning mutants of Tribolium castaneum. Development 1996, 122:805-814. 
4. Pultz MA, Zimmerman KK, Alto NM, Kaeberlein M, Lange SK, Pitt JN, Reeves NL, Zehrung DL: A genetic screen for zygotic embryonic lethal mutations affecting cuticular morphology in the wasp Nasonia vitripennis. Genetics 2000, 154:12131229.

5. Tribolium Genome Sequencing Consortium, Richards S, Gibbs RA, Weinstock GM, Brown SJ, Denell R, Beeman RW, Gibbs $\mathrm{R}$, Beeman RW, Brown SJ, Bucher G, Friedrich M, Grimmelikhuijzen CJ, Klingler M, Lorenzen M, Richards S, Roth S, Schröder R, Tautz D, Zdobnov EM, Muzny D, Gibbs RA, Weinstock GM, Attaway T, Bell S, Buhay CJ, Chandrabose MN, Chavez D, Clerk-Blankenburg KP, Cree A, Dao M, et al:: The genome of the model beetle and pest Tribolium castaneum. Nature 2008, 452:949-955.

6. Tomoyasu Y, Miller SC, Tomita S, Schoppmeier M, Grossmann $D$, Bucher G: Exploring systemic RNA interference in insects: a genome-wide survey for RNAi genes in Tribolium. Genome Biol 2008, 9:R10.

7. Horn C, Schmid BG, Pogoda FS, Wimmer EA: Fluorescent transformation markers for insect transgenesis. Insect Biochem Mol Biol 2002, 32:1221-1235.

8. Lorenzen MD, Kimzey T, Shippy TD, Brown SJ, Denell RE, Beeman RW: piggyBac-based insertional mutagenesis in Tribolium castaneum using donor/helper hybrids. Insect Mol Biol 2007, 16:265-275.

9. Pavlopoulos A, Berghammer AJ, Averof M, Klingler M: Efficient transformation of the beetle Tribolium castaneum using the Minos transposable element: quantitative and qualitative analysis of genomic integration events. Genetics 2004, 167:737-746.

10. Berghammer AJ, Klingler M, Wimmer EA: A universal marker for transgenic insects. Nature 1999, 402:370-371.

11. Savard J, Marques-Souza H, Aranda M, Tautz D: A segmentation gene in Tribolium produces a polycistronic mRNA that codes for multiple conserved peptides. Cell 2006, 126:559569.

12. Galindo MI, Pueyo JI, Fouix S, Bishop SA, Couso JP: Peptides encoded by short ORFs control development and define a new eukaryotic gene family. PLOS Biol 2007, 5:e106.

13. GEKU [http://www.geku-base.uni-goettingen.de]

14. Kittelmann M, Schinko JB, Winkler M, Bucher G, Wimmer EA, Prpic NM: Insertional mutagenesis screening identifies the zinc finger homeodomain 2 (zfh2) gene as a novel factor required for embryonic leg development in Tribolium castaneum. Dev Genes Evol 2009. doi: 10.1007/s00427-0090303-y

15. Schetelig MF, Scolari F, Handler AM, Kittelmann S, Gasperi G, Wimmer EA: Site-specific recombination for the modification of transgenic strains of the Mediterranean fruit fly Ceratitis capitata. Proc Natl Acad Sci USA 2009, 106:1817118176.

16. Fish MP, Groth AC, Calos MP, Nusse R: Creating transgenic Drosophila by microinjecting the site-specific phiC31 integrase mRNA and a transgene-containing donor plasmid. Nat Protoc 2007, 2:2325-2331.

17. Pavlopoulos A, Kontarakis Z, Liubicich DM, Serano JM, Akam $\mathrm{M}$, Patel NH, Averof M: Probing the evolution of appendage specialization by Hox gene misexpression in an emerging model crustacean. Proc Natl Acad Sci USA 2009, 106:1389713902.

Published: 30 December 2009

doi:10.1186/jbiol208

(c) 2009 BioMed Central Ltd 